\title{
Impact of Node Clustering on Power Consumption in WSN
}

\section{A Comparative Study}

\author{
Ala'a H. Makableh, Ghassan Samara \\ Department of Computer Science \\ Zarqa University \\ Zarqa, Jordan
}

\begin{abstract}
Wireless Sensor Networks (WSN) is an important application uses the power of wireless communication to querying the real word. Sensor nodes are battery-driven devices and it have to work as long as possible to gather data. This paper provides a comparative study about using clustering on WSNs and how it helps in saving energy in these sensor nodes. The aim of this study is suggesting a topology to distribute nodes in WSN in a way that enhance overall battery life.
\end{abstract}

\section{Keywords - Wireless Sensor Networks, Clustering, Power Consumption.}

\section{INTRODUCTION}

Wireless Sensor Networks (WSN) is a network of sensor nodes, each node works as station to collect specific data from its environment, organizing these collected data and sent them to central computer as electrical signals wirelessly to be manipulated. In WSN, sensors send these signals either periodically or based on events depending on the goal of censoring.

The importance of WSN comes from its critical using in many applications as in [1] and [2] some of these applications can be divided to fields as follows:

- Military Applications for targeting or detecting the Nuclear, biological and chemical attack.

- Environmental Application like detecting the forests fire, flooding and air or water pollution.

- Health Applications like tracing and monitoring patient in the hospital and drug administration.

- Home applications in which the home machines will be controlled remotely by end user and interacts automatically.

The main component of wireless sensor node consist of:

- Sensing Unit, which is a hardware that responsible about measuring the physical parameters.

- Processing Unit, which is a hardware that process the data, collected by sensor unit and controls the functionality of other components in the sensor node.

- Transceiver Unit, which is a device work as a transmitter and a receiver at the same time.

- Power Unit, which is typically the battery.
Some optional component may be added such as Location Finding System, Power Generator, Mobilizer. A simple structure of sensor node shown in Figure 1.

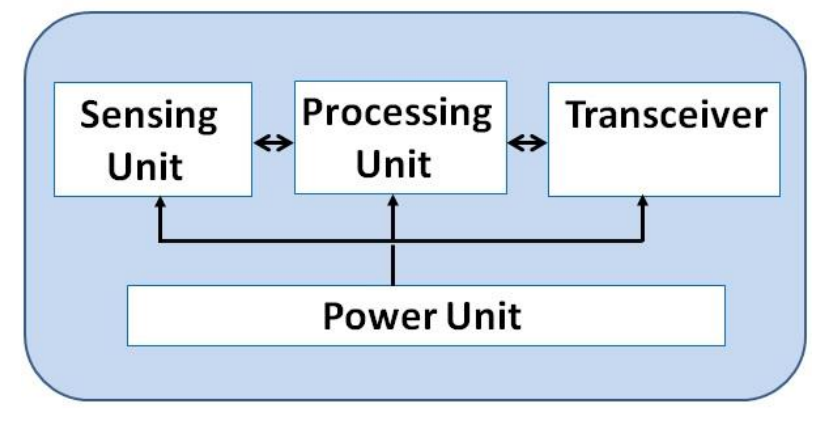

Fig. 1. The main component of wireless sensor node

Since the nodes in WSN are battery-driven and battery is a limited power source, it is important to minimize the energy that the node consume to maximize the overall efficiency of WSN.

The constraints on WSN which come from the limited energy, storage size and processing power leads to take these constraints in account and adapt the wireless network protocols or suggests a new protocols specifically designed for wireless sensor networks to achieve these requirements.

In this paper the power consumption and battery limitation for nodes in WSN is studied, furthermore, this paper will show that clustering can enhance the overall power consumption and provides a topology that exploit the node clustering to enhance power consumption. 
The work is organized as follows. In section II literature review is presented. In section III clustering techniques are discussed and compared. A new model for WSN clustering suggested in section IV. In section V the conclusion presented.

\section{LITERATURE REVIEW}

The previous researches in WSN covered many ideas to solve power consumption and suggested different models. In [3] the power consumption studied from the point of using multihop implementation in WSN to reduce the power consumption for the sensor nodes. In contrary, an opposite result suggested in [4] where a single-hop implementation is used which is simpler in routing protocol and needs a lower communication overhead which is more efficient. Another idea proposed in [5] to serve the applications in environmental science and agriculture by distributing large number of sensor nodes in wireless network which combines a very large radio range with low cost, and transmits only in a single hop mode to get low power consumption. The improvement of hardware and implementing a hardware-based forward error correction scheme is suggested in [6] which gives a better overall energy consumption for the node.

As mentioned in [7] routing protocols classified into seven main categories: Heterogeneity-based Protocols, Locationbased Protocols, Data centric Protocols, Hierarchical Protocols, Mobility-based Protocols, Multipath-based Protocols and QoSbased Protocols. Hierarchical Protocols organizes the nodes in WSN as a small group named cluster.

The cluster has two-level hierarchy of nodes: the first higher is the cluster head nodes collecting data from nodes in its cluster and transmit these data, the second lower is the node members of the cluster which are responsible for collecting the real world data [8]. This saves communication and processing work and also saves energy [9]. The importance of energy efficiency not only in static sensor nodes but also in mobile sensor networks too. One of the modern researches studied the mobile sensor networks and proposed a novel approach to develop an energy efficient routing in [10].

\section{CLUSTERING TECHNIQES:}

A part from clustering architecture of a WSN is treated to minimize the energy consumption by transmitting less data, and this also improves the scalability of the network and the communication bandwidth within the cluster [10]. Some of the energy efficient routing protocols based on clustering are LEACH, HEED, PEGASIS, Hierarchical-PEGASIS, TEEN, APTEEN:

Low Energy Adaptive Clustering Hierarchy (LEACH) [11], this protocol uses the clustering to distribute the energy consumption by dividing network nodes into groups based on data collection. The cluster heads that collects data from the nodes coming under its cluster are randomly selected. This randomly selection helps to prevent energy draining for the same sensor node because head would be changed. The main problem of this protocol is the random selection of cluster head. In the worst case the choose of cluster head nodes may be not distributed fairly, and this effects the data gathering. See figure 2 which shows the LEACH where the cluster heads collects data before transmitting it to the base station (BS) [12].

Hybrid Energy Efficient Distributed clustering approach (HEED) [13] was developed to avoid the problem of random selection of cluster heads selects the cluster heads based on both remained energy level and communication cost.

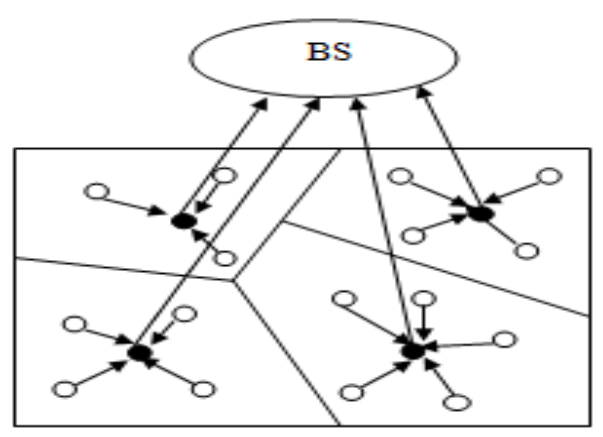

Fig. 2. LEACH Architecture

These protocols are proved energy efficient to WSN with static sensor nodes only and they are untested while the sensor nodes exhibit mobility [10].

Power Efficient Gathering in Sensor Information Systems (PEGASIS), where all the sensor nodes in the network will be arranged to form a chain with a leader node which is responsible for transmitting data to the base station[14]. The data moves from one node to the next until reaching the end of the chain where the leader node lies, the leader node transmits one message to the base station as shown in Figure 3 [15]. The main problem of this protocol is the long delay.

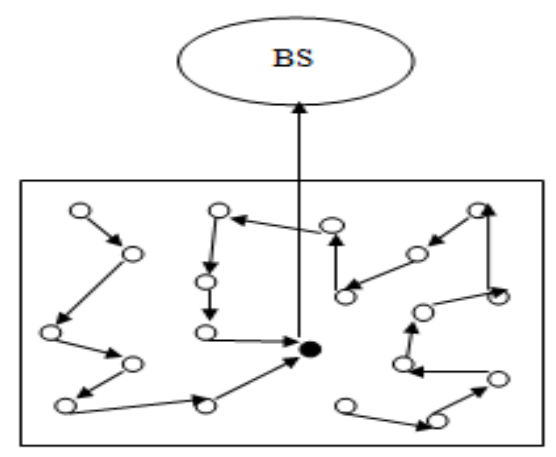

Fig. 3. PEGASIS Architecture

Hierarchical-PEGASIS is an enhancement on PEGASIS, to reduce transmission delay [16].

Threshold Sensitive Energy Efficient Sensor Network Protocol (TEEN) is a hierarchical clustering based protocol in which nodes react with the changes in the environment. After 
cluster formation, the cluster head broadcasts two thresholds to the sensor nodes namely hard threshold and soft threshold. Hard threshold permits the sensor nodes to send data only when the attribute sensed by them is in the range of interest. The soft threshold will reduce the data transmission if there is no or little change in the value of sensed attribute. In order to control the data transmissions, both thresholds can adjust [17].

The Adaptive Threshold sensitive Energy Efficient sensor Network protocol (APTEEN) is an extension to TEEN. Clustering in APTEEN and TEEN protocols is done in two levels of cluster heads: the first level collects data from set of simple nodes and the second level cluster heads gets data from cluster heads in the first level and its simple nodes to transmit it to base station as shown in Figure 4.

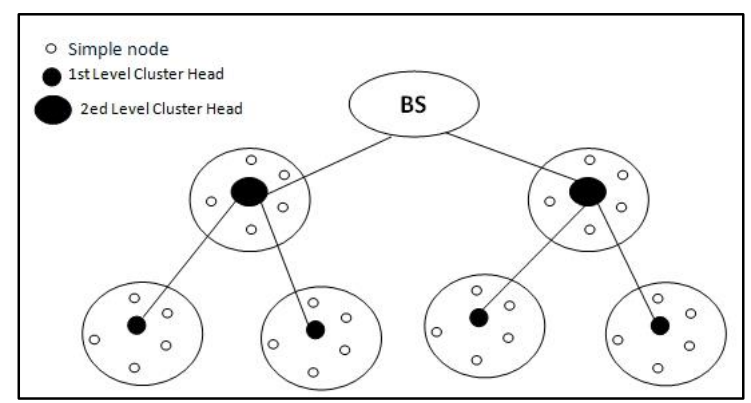

Fig. 4. Hierarchical Clustering in TEEN

A summary to these protocols can be as follows [13]:

LEACH, TEEN, APTEEN and PEGASIS have almost similar features and fixed infrastructure. LEACH, TEEN, APTEEN are cluster based routing protocols but PEGASIS is a chain-based routing protocol. The performance of APTEEN is intermediate between TEEN and LEACH in the field of power consumption of the network. PEGASIS avoids the overhead of cluster formation of LEACH, but it needs dynamic topology adjustment. PEGASIS adds excessive delay for distant nodes on the chain.

\section{PROPOSED SYSTEM}

Based on nature we propose a perfect hexagon clustering system to the WSN. This system takes the advantages of hexagon shape like what the bee do in beehive to get maximum amount of space with a minimal amount of material.

When the area of the cluster of WSN is divided into a grid of hexagon the resulted grid will have no gaps and it covers the maximum area unlike the other shapes such as the circle or octagon create gaps and the triangle or square makes the area smaller as sown in figure 5 .

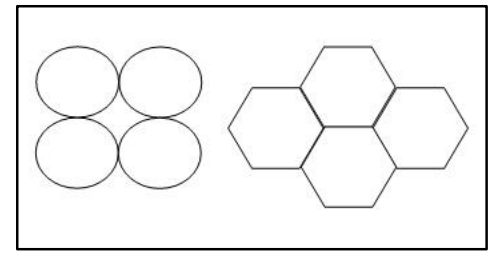

Fig. 5. No gaps in hexagon grid

In the cluster, each hexagon will be called cluster cell. The cluster cell will have sensor node in the central, this node responsible about sensing within its cell area. By applying this to all cluster cells the whole cluster will cover the maximum area and the distances between these nodes will be minimized to get efficient communication with low power consumption within the nodes of the same cluster. Figure 6 shows the architecture of such system.

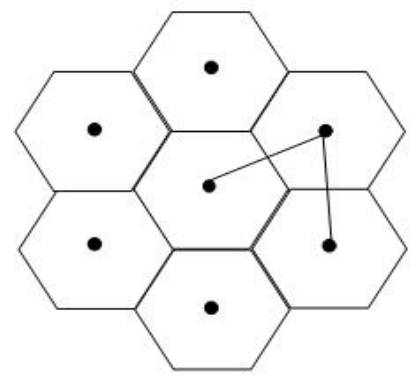

Fig. 6. a perfect hexagon cluster with centric sensor nodes

\section{CONCLUSION}

In WSN it is still important to maximize the network nodes efficiency with minimization to the power consumption. This paper studied the clustering protocols and suggested a new clustering model. This model enhances the cluster efficiency by maximizing the sensing area with better communication distances to minimize the power consumed in communication between nodes.

\section{REFERENCES}

[1] John A. Stankovic, Anthony D. Wood, Tian He, "Realistic Applications for Wireless Sensor Networks", Theoretical Aspects of Distributed Computing in Sensor Networks,Springer Verlag, 2010.

[2] I. F. Akyildiz, W. Su, Y. Sankarasubramaniam, and E. Cayirci, "Wireless Sensor Networks: A Survey", Elsevier Computer Networks, March 2002.

[3] J. M. Rabaey, J. Ammer, T. Karalar, S.Li, B. Otis, M. Sheets, T. Tuan, "Pico Radios for wireless sensor networks-the next challenge in ultra-low power design, International Solid-State Circuits Conference (ISSCC), 2002. 
[4] Zhong, L.C.; Rabaey, J.M.; Wolisz, “ Does proper coding make single-hop wireless sensor networks reality: the power consumption perspective", Wireless Communications and Networking Conference (WCNC) IEEE, 2005.

[5] C. Huebner, R. Cardell-Oliver, S. Hanelt, T. Wagenknecht, A. Monsalve, "Long range wireless sensor networks with transmitonly nodes and software defined receivers", Canadian Society of Safety Engineering (CSSE) Technical Report, July 2010.

[6] Andreas Brokalakis, Ioannis Papaefstathiou, "Using hardwarebased forward error correction to reduce the overall energy consumption of WSNs", Wireless Communications and Networking Conference (WCNC) IEEE, 2012.

[7] Parul Tyagi, Surbhi Jain, "Comparative Study of Routing Protocols in Wireless Sensor Network", International Journal of Advanced Research in Computer Science and Software Engineering, 2012.

[8] Vivek katiyar, Narottam chand, Surender soni, "A Survey on Clustering Algorithms for Heterogeneous Wireless Sensor Networks", International Journal of Advanced Networking and Applications, 2011.

[9] Ameer Ahmed Abbasi, Mohamed Younis, "A survey on clustering algorithms for wireless sensor networks", IEEE Communication Magazine, 2007.

[10] B.Baranidharan, B.Shanthi, "A Survey on Energy Efficient Protocols for Wireless Sensor Networks", International Journal of Computer Applications (0975 - 8887), 2010.

[11] W. B. Heinzelman, A. P. Chandrakasan, H. Balakrishnan, "Application specific protocol architecture for wireless micro sensor networks", IEEE Transactions on Wireless Networking 2002.

[12] Anjali Bharti, Kanika Sharma, "Comparative Study of Clustering based Routing Protocols for Wireless Sensor Network", International Journal of Computer Applications (0975 - 8887), 2013.

[13] O. Younis, S. Fahmy, "HEED: A Hybrid Energy-Efficient Distributed clustering approach for Ad Hoc sensor networks", IEEE Transactions on Mobile Computing 3, 2004.

[14] Lindsey, S. Raghavendra, C. S., "PEGASIS: Power Efficient gathering in sensor information systems", Proceedings of IEEE Aerospace Conference, 2002.

[15] Jisoo Shin, Changjin Suh, "CREEC: Chain Routing with Even Energy Consumption", IEEE Journal of Communications and Networks, 2011.

[16] S. Lindsey, C. S. Raghavendra, K. Sivalingam, "Data gathering in sensor networks using the energy*delay metric", IEEE International Parallel \& Distributed Processing Symposium (IPDPS) Workshop on Issues in Wireless Networks and Mobile Computing, 2001.

[17] Manjeswar, A. Agrawal, D. P., "TEEN: A protocol for enhanced efficiency in wireless sensor networks", International Workshop on Parallel and Distributed Computing Issues in Wireless Networks and Mobile computing, 2001. 